\title{
SofiaFala: Software Inteligente de Apoio à Fala
}

\author{
Pedro Henrique D’Almeida Giberti Rissato* \\ Alessandra Alaniz Macedo ${ }^{\dagger}$ \\ pedro.rissato@usp.br \\ ale.alaniz@usp.br \\ Departamento de Computação e Matemática - FFCLRP -USP \\ Ribeirão Preto, São Paulo
}

\begin{abstract}
Approximately 2.7 million Brazilians may have some speech disorder, according to the Brazilians Institute of Geography and Statistics. Language therapies usually employ speech exercises at home for people with speech disorders without close specialized supervision. There are fundamental movements. Assistive technology and machine learning can support the development of speech pronunciation practice systems. SofiaFala is a mobile application that supports prescription, monitoring, execution, and evaluation of speech therapies. It also supports speech therapist data analysis for the outside clinical environment. In the two last years, 1,400 speech therapists have required access to the SofiaFala.
\end{abstract}

\section{KEYWORDS}

Speech \& Pattern Recognition, Machine Learning, Speech Disorder

\section{INTRODUÇÃO}

Quando Sofia nasceu, sua mãe se deu conta de que ela necessitaria de diferentes tipos de recursos terapêuticos para ajudá-la, uma vez que foi diagnosticada com Síndrome de Down (SD) ${ }^{1}$. Sofia precisaria de acompanhamento fonoaudiológico precocemente para comer adequadamente, desenvolver a fala, evitar bullying na adolescência, e alcançar condições de empregabilidade e bem-estar na vida adulta. A mãe de Sofia buscou apoio em parceiros na universidade para criar uma tecnologia de apoio a fala que fosse adequada à realidade das famílias brasileiras, considerando que as tecnologias existentes não cumpriam com o acompanhamento necessário. Nesse contexto, surgiu o grupo de pesquisa SofiaFala ${ }^{2}$, formado por pais, educadores, psicólogos, médicos, fonoaudiólogos e cientistas da computação.

\footnotetext{
${ }^{*}$ Mestrando do Departamento de Computação e Matemática da FFCLRP da Universidade de São Paulo (USP), Campus Ribeirão Preto. Lattes: http://lattes.cnpq.br/9400608160057217

†Professora Associada do Departamento de Computação e Matemática da FFCLRP da Universidade de São Paulo (USP), Campus Ribeirão Preto. Lattes: http://lattes.cnpq.br/2407277993285186

${ }^{1}$ A Síndrome de Down (SD) é uma alteração genética advinda de uma cópia adicional do cromossomo 21. A pessoa com SD é acometida por uma série de alterações fenotípicas, que impactam a aparência sem necessariamente apresentarem demais complicações, intelectuais e físicas [6]. Dentre as modificações físicas apresentadas, a diminuição do tônus muscular dos músculos da face e da fala, contribuem diretamente para disformidade da fala, incluindo desordens auditivas em decorrência do atraso no desenvolvimento da linguagem [2].

${ }^{2} \mathrm{Em}$ http://dcm.ffclrp.usp.br/sofiafala existem informações de acesso ao aplicativo, vídeos demonstrativos da execução, reportagens, etc.
}

In: XX Workshop de Ferramentas e Aplicações (WFA 2021), Minas Gerais, Brasil. Anais Estendidos do Simpósio Brasileiro de Sistemas Multimídia e Web (WebMedia). Porto Alegre: Sociedade Brasileira de Computação, 2021.

(C) 2021 SBC - Sociedade Brasileira de Computação.

ISSN 2596-1683
Apesar da motivação para SD, o público-alvo do SofiaFala é formado por pessoas acometidas por Transtornos de Fala (TF), os quais podem decorrer também de deficiência intelectual e de audição, Transtorno Espectro Autista (TEA), sequelados de AVC, idosos, além das disartrias e apraxias na infância. A Organização Mundial da Saúde informa que, no mundo, uma em 160 crianças têm TEA ${ }^{3}$, uma em mil têm $\mathrm{SD}^{4}$, e de 10 a $30 \%$ das crianças têm TF. Uma pesquisa do Centro Integrado de Inclusão e Reabilitação de Ribeirão Preto (SP) constatou que, em 500 atendimentos em Fonoaudiologia, $65 \%$ das crianças entre 3 e 8 anos têm TF [10]. Segundo o Censo Demográfico de 2010 do Instituto Brasileiro de Geografia e Estatística $^{5}$, quase $25 \%$ da população do país, 45 milhões de brasileiros, têm algum tipo de deficiência de visão, audição, motora ou intelectual. Dessa população, $5 \%$ têm deficiência auditiva e $1 \%$ deficiência mental ou intelectual. Além das apraxias na infância e dos sequelados, cerca de 3 milhões de pessoas têm TF. É muito importante que o governo e a sociedade pensem em ações para incluir os brasileiros que tem ou desenvolveram TF em alguma fase da vida.

Para tratar TF, os fonoaudiólogos estabelecem diversos exercícios de treinamento com o intuito de fortalecer os músculos orofaciais, que são utilizados para produzir os sons e palavras [4]. Entretanto, apenas uma parcela dos treinamentos são acompanhadas fisicamente pelo profissional fonoaudiológico em consultório. Grande parte da prática é realizada na casa do paciente. A carência do acompanhamento profissional próximo, distrações externas à realização dos treinos, aliadas à falta de periodicidade, acarretam problemas na prática correta dos exercícios. Outro problema declarado pelos terapeutas é o fato de não conseguirem saber exatamente como os exercícios de treinamento prescritos foram realizados.

Tecnologias assistivas aliadas às técnicas de Aprendizado de Máquina (AM) podem ser usadas para (i) reconhecer padrões fonoaudiológicos desejados, (ii) promover uma análise objetiva dos resultados e (iii) suportar sessões de terapia da fala, de modo a serem consideradas uma extensão virtual do fonoaudiólogo. Esse tipo de esforço pode prover uma forma lúdica-educacional de engajar paciente no treino de fala. Motivados por essas possibilidades, o aplicativo "Software Inteligente de Apoio à Fala" (SofiaFala) foi desenvolvido para auxiliar o fonoaudiólogo na intervenção terapêutica para evolução da fala da pessoa com TF.

\section{ARQUITETURA E FUNCIONALIDADES}

O desenvolvimento do SofiaFala originou uma aplicação móvel para auxiliar treinos da fala prescritos por fonoaudiólogos para pessoas

\footnotetext{
${ }^{3}$ https://www.paho.org/pt/topicos/transtorno-do-espectro-autista

${ }^{4}$ https://apps.who.int/iris/handle/10665/263361

${ }^{5}$ https://cnae.ibge.gov.br/
} 
com Transtorno de Fala (TF). O aplicativo possui dois módulos: o Módulo Fonoaudiólogo (MF) e o Módulo Paciente (MP), que funcionam de forma integrada e sincronizada. O MF auxilia na preparação e prescrição do treinamento da fala composto por movimentos orofaciais. O MF também permite cadastrar pacientes, produzir mídias, que demonstrem como realizar um exercício, e visualizar relatórios construídos, a partir dos dados recebidos pelo MP. Esses relatórios oferecem aos terapeutas oportunidades de acompanhar de perto o desempenho de seus pacientes com avaliações quantitativas e qualitativas. Os módulos MP e MF rodam em dispositivos móveis usando o sistema operacional Android desde a API $21^{6}$, utilizando principalmente a linguagem fava e complementarmente Python e $\mathrm{C++}$. A Figura 1 ilustra a arquitetura do MP. O MF segue a mesma arquitetura, diferenciando apenas os serviços e sub-rotinas das camadas de modelo e de apresentação.

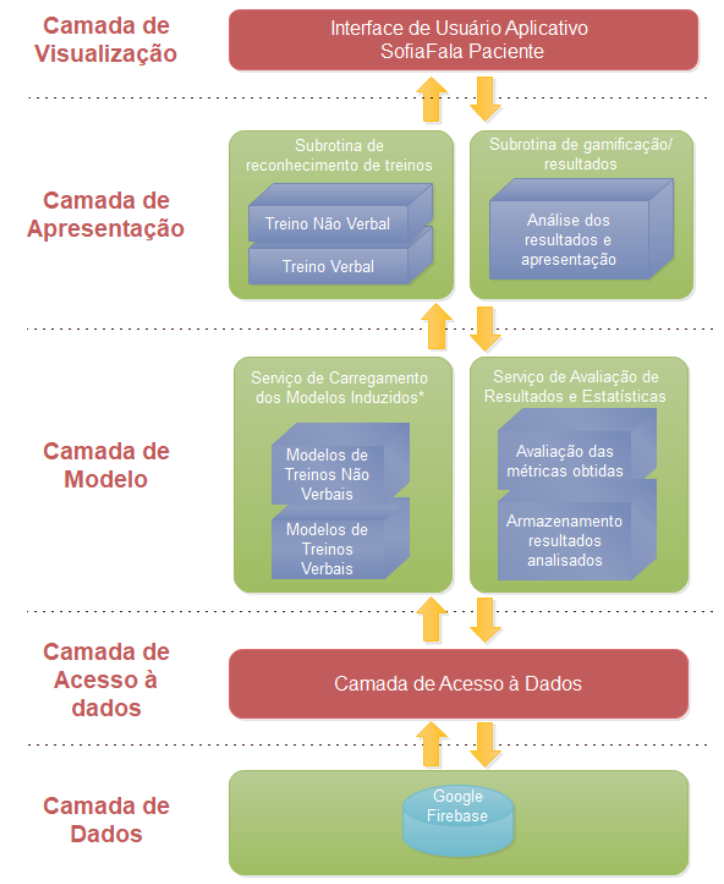

Figura 1: Arquitetura do SofiaFala: Módulo Paciente. ${ }^{7}$

O padrão de projeto Model-View-Presenter (MVP) [7] foi adotado para organizar as trocas de informações entre a camada de dados dos pacientes e a interface de usuário. Os comandos dos usuários são transferidos pela View, via interface, para o Presenter, que seguindo o modelo definido no Model age, buscando as informações na base de dados e enviando para as Views preparadas para serem exibidas.

\footnotetext{
${ }^{6}$ Disponível em https://source.android.com/setup/start/build-numbers. Segundo a IDE Android Studio v2020.3.1, a API 21 de desenvolvimento do Android cobre 94,1\% de todos os dispositivos móveis, tablets e celulares disponíveis no mercado. Consulta realizada em 10/08/2021.

${ }^{7}$ Considerando o serviço de treino não-verbal de bico-sorriso, parte da implementação para analisar os frames da câmera foi feita em C++ e no treino beijo, estalo de língua e sopro, o software development kit (SDK) Chaquopy foi utilizado. Ambas atuam na camada de bibliotecas nativas $\mathrm{C} / \mathrm{C}++$ da arquitetura do Android disponível em https://developer.android.com/guide/platform?hl=pt-br. Modelo adaptado de https://www.edrawsoft.com/pt/architecture-diagram.html.
}

Para armazenar os dados oriundos de ambos os módulos, foi escolhido o Firebase Realtime Database, um banco de dados do tipo NoSQL [9] baseado em documentos que armazenam objetos em notação fava Script ou JSON contendo dados semi-estruturados. Esse banco é ofertado pela empresa Google, nos formatos gratuito e pago $^{8}$, na plataforma Firebase ${ }^{9}$, sendo totalmente baseado em computação na nuvem em um modelo cliente-servidor.

O aplicativo SofiaFala é capaz de reconhecer os movimentos da face que produzem sons verbais (consoantes, vogais, sílabas ou palavras e frases inteiras) e não-verbais (sopros, estalos de língua, beijos e movimentos bico-sorriso), por meio da análise de vídeo e de áudio. No caso de sons verbais, a biblioteca SpeechRecognizer do próprio Android ${ }^{10}$ foi utilizada para reconhecer textualmente as palavras pronunciadas, em conjunto com um algoritmo próprio para prevenir que erros fonéticos sejam reconhecidos como a palavra de interesse. Os treinos de sons não-verbais desenvolvem músculos essenciais no suporte da fala. Para esses treinos, foram desenvolvidos dois modelos. Um modelo, denominado ANA (Approach for Non Articulatory Sounds) [8], reconhece sons não-verbais, em especial, os emitidos por beijo, sopro e estalo de língua. O modelo ANA deu suporte a um estudo piloto do SofiaFala que indicou a eficácia dos métodos usados em sons não-verbais. O outro modelo [5] é baseado em algoritmo próprio que em conjunto com marcadores importantes na face humana, denominados de landmarks ${ }^{11}$, é capaz de avaliar se o movimento de bico-sorriso é realizado corretamente, respeitando um espaço temporal e critérios pré-estabelecidos, transcritos em algoritmo, conforme avaliação da equipe de fonoaudiólogos vinculados ao projeto SofiaFala.

\section{USO EM TREINOS}

No dia a dia, fonoaudiólogos recebem pacientes e realizam anamneses iniciais, e de acordo com o nível de dificuldades fonoaudiológicas, estabelecem a terapêutica, a ser seguida pelo paciente. Para definir esse nível, eles usualmente aplicam testes ABFW [1] e AMIOFE [3].

O SofiaFala tem dois atores principais, o paciente e o profissional fonoaudiólogo. Usando seu MF, o profissional cadastra o paciente e fornece uma senha única para o paciente baixar e acessar o MP do aplicativo. Esse procedimento foi adotado para garantir que o SofiaFala seja utilizado mediante o acompanhamento profissional de um fonoaudiólogo. A Figura 2a ilustra as informações necessárias que o profissional deverá indicar para transportar a terapêutica para a forma de treinos no aplicativo.

Ao cadastrar os treinos, o fonoaudiólogo pode estabelecer a quantidade de repetições diárias que o paciente deve realizar, a quantidade de vezes que as repetições devem ocorrer dentro dos treinos, data de início e término, e, inclusive, quais dias da semana os exercícios devem ser realizados. Essas configurações pretendem englobar as diferentes possibilidades terapêuticas, além de evitar, por exemplo, que o paciente realize todo o treino logo antes de uma

\footnotetext{
${ }^{8} \mathrm{O}$ projeto SofiaFala utiliza a versão gratuita do serviço que permite armazenar até 1 gigabyte em dados, além de 50.000 leituras, 20.000 escritas e exclusões diariamente. Disponível em https://firebase.google.com/docs/firestore/quotas. Consultado em 11/08/2021. ${ }^{9}$ Disponível em https://firebase.google.com/docs/database.

${ }^{10}$ Disponível em https://developer.android.com/reference/android/speech/SpeechRecognizer

${ }^{11} \mathrm{O}$ reconhecimento dos landmarks foi implementado por meio da biblioteca dlib portada para Android disponível em https://github.com/tzutalin/dlib-android.
} 


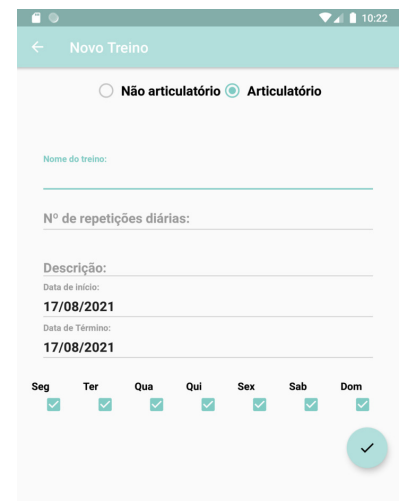

(a) Tela de cadastro de treino no MF. (b) Tela de seleção do treino do MP.

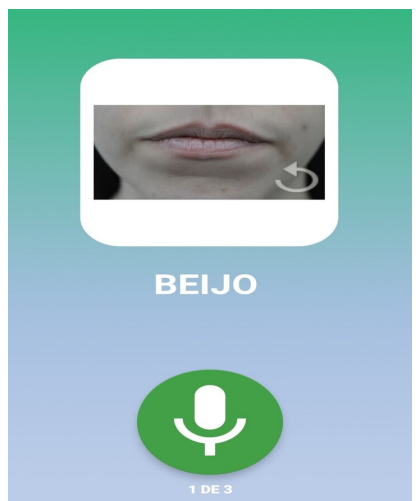

(c) Treinamento de movimento não-verbal do tipo beijo no MP.

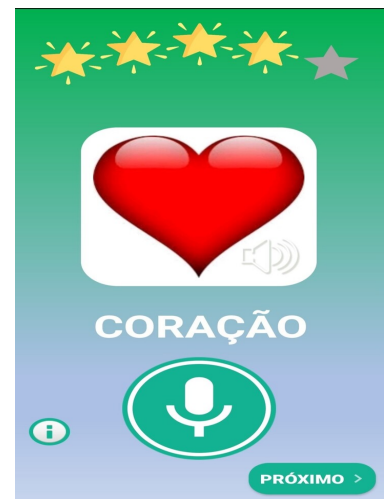

(d) Treinamento da palavra "coração", pronunciada como “oração" no MP.

Figura 2: Telas do Aplicativo SofiaFala: Módulo Paciente (MP) e Módulo Fonoaudiólogo (MF).

consulta com o fonoaudiólogo. Também é possível ao fonoaudiólogo, cadastrar novas palavras para o treinamento desejado, assim como escolher uma imagem de referência contextualizada com a realidade do paciente, para apresentação durante o treino. Além disso, o profissional pode gravar áudios de exemplo, ou, escolher dentre as 556 palavras previamente cadastradas com os fonemas mais relevantes para treinamento da fala.

Após o cadastro, o treino é disponibilizado em tempo real ao paciente, que deve seguir a conduta terapêutica prescrita. Durante todo o treinamento do paciente, o fonoaudiólogo possui acesso a métricas do histórico do treino do paciente no MF do SofiaFala, como desempenho máximo, médio e mínimo e frequência da realização dos treinos, agrupadas por períodos selecionáveis. Assim, utilizando métricas objetivas, extraídas dos próprios modelos de avaliação e áudios gravados, é possível realizar uma análise detalhada da evolução do paciente bem como da sua adesão e da eficácia da terapêutica aplicada.

O paciente acessa o Módulo Paciente (MP) e encontra as sessões de treino disponíveis para iniciá-las. A Figura $2 \mathrm{~b}$ ilustra a tela de acesso do paciente. Nessa tela, o paciente pode acessar todos os treinos disponibilizados pelo fonoaudiólogo, separados por tipos de treinos e agrupados por dias da semana. A interface de usuário é simples e intuitiva para auxiliar os pacientes, principalmente crianças ou pessoas com alguma deficiência intelectual.

\subsection{Treinos Não-Verbais}

Os treinos não-verbais (como beijo, estalo de língua, sopro ou bicosorriso) fortalecem músculos e estruturas orofaciais, a fim de propiciar a produção de fonemas, sílabas, palavras e frases. A Figura 2c ilustra a tela inicial de treinos não-verbais para reconhecimento de um beijo, por exemplo.

Ao iniciar o treino, um vídeo realizado por uma fonoaudióloga é executado, ensinando a realização do movimento da maneira mais adequada. O paciente deve apertar o botão com o símbolo de microfone para iniciar a gravação do áudio do movimento de interesse, após um tempo limite estabelecido ou apertando novamente o botão, o paciente pára a gravação. Automaticamente, o SofiaFala envia o áudio para extração das características e análise do modelo de Aprendizado de Máquina. Em seguida, uma tela intermediária apresenta cinco estrelas, as quais são preenchidas de acordo com a nota atribuída pelo modelo, como na Figura 2d. As cinco estrelas são preenchidas quando um limiar estabelecido for superado; e três estrelas são preenchidas para qualquer valor abaixo desse limiar. Essa decisão de projeto foi tomada para incentivar o uso do aplicativo, uma vez que após avaliações iniciais com crianças, percebeu-se a necessidade de reforço positivo para estimular a prática.

Após a apresentação da nota, o paciente retornará à tela de treino, caso existam mais repetições a realizar do mesmo tipo ou de outro movimento ainda no mesmo treino. Na Figura 2c, pode-se observar o número de repetições realizadas e o número restante. Caso não existam mais repetições, o usuário é retornado à tela principal, o treino é considerado finalizado e os dados são disponibilizados, em tempo real, para o fonoaudiólogo que acompanha o paciente.

Os mesmos fluxos de atividades utilizados no treino não-verbais do beijo, do estalo de língua e do sopro são aplicáveis para o treino não-verbal do tipo bico-sorriso. Contudo, tratando-se agora de análise de vídeo, a interface de treino, como da Figura 2c, possui um ícone da câmera. Ao clicar no ícone, a câmera frontal do dispositivo móvel é ligada para captar o movimento. Caso seja a primeira vez da realização do treino, o paciente será redirecionado para tirar uma foto de rosto inteiro para extração de distâncias envolvendo a boca e os olhos do paciente ${ }^{12}$ para comparações com o modelo preditivo. $\mathrm{Na}$ interface de treino de bico-sorriso, existe um botão no canto inferior esquerdo para selecionar a câmera traseira do dispositivo.

\subsection{Treinos Verbais}

A Figura $2 \mathrm{~d}$ apresenta a tela inicial do treino verbal. No treino verbal, o paciente poderá ouvir a palavra a ser repetida ao pressionar o símbolo de alto falante. Também existe uma ilustração da palavra de

\footnotetext{
${ }^{12}$ Esses dados não permitem qualquer tipo de identificação do paciente, são meramente distâncias Euclidianas entre pontos de interesse selecionados na face do paciente para comparação no algoritmo que detecta se o paciente está realizando um bico e/ou um sorriso. Os dados dessas distâncias são armazenados apenas no dispositivo móvel do próprio paciente.
} 
interesse, para apresentação multimodal da palavra a ser repetida pelo paciente. Ao clicar no microfone, inicia-se a captação da palavra de interesse, ao final, uma nota é atribuída e convertida em exibição com as estrelas acima da imagem, disponibilizando o botão para a próxima palavra ou encerramento do treino.

\section{RESULTADOS}

O aplicativo SofiaFala foi oficialmente distribuído, a partir de Julho de 2019, sendo de uso restrito de fonoaudiólogos e seus pacientes. Por essa razão, um cadastro prévio é exigido no site do projeto. Até $11 / 08 / 2021$, foram solicitados 1391 acessos únicos ao SofiaFala dos quais 1253 foram liberados e 138 foram negados por não partirem de fonoaudiólogos ou por impedimentos junto ao Conselho Regional de Fonoaudiologia. Dos aprovados, 559 efetivamente realizaram o cadastro no aplicativo. Essa discrepância entre o número de autorizações e de efetivações sugere a possibilidade de algum tipo de dificuldade entre a autorização e o cadastramento no aplicativo, o que deve ser motivo de investigação em um futuro próximo.

Os dez estados do Brasil em que os fonoaudiólogos mais solicitaram acessos foram respectivamente SP (508), MG (164), RJ (126), PR (135), RS (70), SC (72), DF (49), BA (44), GO (38) e ES (25). Ao solicitarem acesso, os fonoaudiólogos responderam como tomaram conhecimento do SofiaFala, 78,3\% (1089) informaram que conheceram o SofiaFala via WhatsApp, 11,6\% (161) por meio de reportagem na internet e outros 10,0\% por meio de Facebook, Instagram, reportagem na televisão e $0,1 \%$ não responderam.

Os fonoaudiólogos cadastraram 803 pacientes. Para esses pacientes, foram cadastrados 1248 treinos, sendo 412 do tipo verbal para o reconhecimento de sons de palavras inteiras, 836 do tipo não-verbal para reconhecimento de beijo (394), sopro (108) e estalo de língua (160) mediante análise do som emitido e 174 do tipo bicosorriso, por meio da análise dos vídeos dos pacientes realizando os movimentos de interesse.

\section{CONCLUSÃO E TRABALHOS FUTUROS}

Distúrbios da comunicação decorrem de alterações da fala, linguagem, e da audição. Fala ou linguagem estão intimamente relacionadas a integridade neuromuscular, do sistema sensorial; as influências do meio e das condições psicológicas, e a perda de audição estão mais relacionadas a doenças, idades ou fatores externos como exposição a ruídos. Portanto, habilidades de comunicação podem ser degradas em processos naturais ou patológicos como envelhecimento, doenças, síndromes ou acidentes que podem levar a uma série de problemas e/ou deficiências de médio a longo prazo.

O grupo SofiaFala desenvolveu e busca aperfeiçoar uma ferramenta de uso pessoal criada especificamente para melhorar a capacidade funcional de uma pessoa em termos de fala, e consequentemente, de comunicação para que ela possa se integrar na sociedade. Com isso, busca-se equiparação de oportunidades para essas pessoas. $\mathrm{O}$ aplicativo SofiaFala é uma tecnologia assistiva treinamento da fala,a qual é dividida nos Módulos Paciente e Fonoaudiólogo, disponíveis sob o acordo de licença de usuário final ${ }^{13}$. $\mathrm{O}$ aplicativo agrega métricas aos trabalhos dos fonoaudiólogos elevando o potencial de análise e terapêutica dos seus pacientes.

\footnotetext{
${ }^{13}$ Disponível em https://dcm.ffclrp.usp.br/sofiafala/eula.php.
}

O SofiaFala contempla os Objetivos de Desenvolvimento Sustentável (ODS) da Agenda 2030 da ONU, uma vez que a deficiência é referenciada nos ODS, especificamente em partes relacionadas à educação, crescimento e emprego, desigualdade, acessibilidade, bem como coleta de dados e monitoramento dos ODS.

Em termos de trabalhos futuros, pretende-se finalizar a sistematização da avaliação de usabilidade do SofiaFala e escrever um artigo científico com a discussão dos resultados. Outras atividades futuras serão:

- ainda em 2022, o desenvolvimento de uma versão para o sistema operacional $i O S$, correções para o sistema atual, Android e desenvolvimento de novas funcionalidades;

- em 2022, uma nova avaliação da eficácia da aplicação, adesão e evolução do tratamento dos pacientes do SofiaFala;

- em 2023, a distribuição da nova versão.

Os trabalhos futuros serão desenvolvidos com o apoio de parceiros como Ribdown ${ }^{14}$, FADA ${ }^{15}$, Faculdade de Medicina de Ribeirão Preto/SP, Faculdade de Odontologia de Bauru/SP e do Centro Integrado de Inclusão e Reabilitação (CIIR) de Ribeirão Preto/SP.

\section{AGRADECIMENTOS}

Os autores gostariam de agradecer a todos os participantes e colaboradores do projeto SofiaFala, especialmente a Marinalva e Sofia Dias Soares, a Profa Dra Patrícia Mandrá, Vinicius Souza, Leandro Persona, Fernando Meloni, Bianca Bortolai, e Renato Bulcão de Freitas Neto, que colaboraram para o desenvolvimento do projeto. Os autores também agradecem ao CNPq (442533/2016-0) e FAPESP (2016/13206-4) pelo financiamento do projeto apresentado.

\section{REFERÊNCIAS}

[1] CRF de Andrade, Débora Maria Befi-Lopes, Fernanda Dreux Miranda Fernandes, and Haydée Fiszbein Wertzner. 2004. ABFW: teste de linguagem infantil nas áreas de fonologia, vocabulário, fluência e pragmática. São Paulo: Pró-Fono (2004).

[2] T. M. M. F. BARBOSA, G. R. G. RABELO, I. L. B. LIMA, and I. C. DELGADO. 2015. Avaliação da linguagem na Síndrome de Down: análise de protocolos desenvolvidos em extensão universitária. In XXIII CONGRESSO BRASILEIRO DE FONOAUDIOLOGIA. 6119.

[3] Cláudia Maria de Felício, Gislaine Aparecida Folha, Alice Stahl Gaido, Márcio de Mendonça Mancine Dantas, and Paulo Mazzoncini de Azevedo-Marques. 2014. Computerized protocol of orofacial myofunctional evaluation with scores: usability and validity. CoDAS 26, 4 (July 2014), 322-327. https://doi.org/10.1590/ 2317-1782/201420140021

[4] Vanessa Giacchini, Aline Tonial, and Helena Mota. 2013. Aspectos de linguagem e motricidade oral observados em crianças atendidas em um setor de estimulação precoce. Distúrbios da Comunicação 25, 2 (2013). https://revistas.pucsp.br/dic/ article/view/16478

[5] Fernando Meloni, Bianca Sicchieri, Patricia Mandrá, Renato Bulcão-Neto, and Alessandra Alaniz Macedo. 2021. A Nonverbal Recognition Method to Assist Speech. In 2021 IEEE 34th International Symposium on Computer-Based Medical Systems (CBMS). 360-365. https://doi.org/10.1109/CBMS52027.2021.00111

[6] David Patterson. 2009. Molecular genetic analysis of Down syndrome. Human Genetics 126, 1 (01 Jul 2009), 195-214. https://doi.org/10.1007/s00439-009-0696-8

[7] Mike Potel. 1996. MVP: Model-View-Presenter the Taligent programming model for C++ and Java. Taligent Inc (1996), 20.

[8] Francisco Carlos M. Souza, Alinne C. Correa Souza, Carolina Y. V. Watanabe, Patricia Pupin Mandrá, and Alessandra Alaniz Macedo. 2019. An Analysis of Visual Speech Features for Recognition of Non-articulatory Sounds using Machine Learning. International fournal of Computer Applications 177, 16 (Nov 2019), 1-9. https://doi.org/10.5120/ijca2019919393

[9] Michael Stonebraker. 2010. SQL Databases v. NoSQL Databases. Commun. ACM 53, 4 (April 2010), 10-11. https://doi.org/10.1145/1721654.1721659

[10] D. L. ZAURA. 2018. Perfil de usuários atendidos em serviço de diagnóstico de linguagem de média complexidade. Trabalho de Conclusão de Curso.

\footnotetext{
${ }^{14}$ www.ribdown.org.br

${ }^{15}$ www.familiadiversidade.com.br
} 